\title{
La autoestima y sus vicisitudes (otra vuelta de tuerca) ${ }^{1}$
}

\author{
Roberto Arendar ${ }^{2}$ \\ IARPP, Buenos Aires, R. Argentina
}

\begin{abstract}
Este trabajo amplía uno anterior de 2014. Apoyado en tres ilustraciones clínicas el autor concluye que: 1) los trastornos de la autoestima son ubicuos; 2) hemos de prestar atención a las convicciones irracionales de defectuosidad e inferioridad que las sustentan; 3) hemos de explorar los distintos contextos intersubjetivos pasados y presentes que han contribuido a forjar la baja autoestima; 4) las configuraciones relacionales que sustentan esa baja autoestima tienen carácter recurrente y 5) el avance en la autoestima es discontinuo, se avanza y se retrocede, y es la tendencia a su mejora lo que ha de importarnos.
\end{abstract}

\section{Palabras clave: Auto-estima, Psicoanálisis Relacional}

This work expands an earlier one of 2014. Supported by three clinical illustrations, the author concludes that: 1) self-esteem disorders are ubiquitous; 2 ) we must pay attention to the irrational convictions of defectiveness and inferiority that sustain them; 3 ) we must explore the different past and present intersubjective contexts that have contributed to forge low self-esteem; 4 ) the relational configurations that sustain that low self-esteem have a recurring character and 5 ) the advance in selfesteem is discontinuous, progresses and recedes, and it is the tendency to its improvement that has to matter to us.

Key Words: Self-esteem, Relational Psychoanalysis.

English Title: Self-esteem and its vicissitudes (a review and new ideas).

\section{Cita bibliográfica / Reference citation:}

Arendar, R. (2018). La autoestima y sus vicisitudes (otra vuelta de tuerca). Clínica e Investigación Relacional, 12 (2): 260-267. [ISSN 1988-2939] [Recuperado de www.ceir.info ] DOI: $10.21110 / 19882939.2018 .120204$

\footnotetext{
${ }^{1}$ Trabajo leído como conferencia en Ágora Relacional, Instituto de Psicoterapia Relacional, Madrid, el 1 de Junio de 2018.

${ }^{2}$ Roberto Arendar es Médico y Psicoanalista. Miembro de IARPP. Ex - Miembro de la Asociación Psicoanalítica de Buenos Aires (APdeBA, IPA). Correo electrónico: robertoarendar@fibertel.com.ar 


\section{Introducción}

Antes de ponerme a escribir un trabajo naturalmente tiendo a preguntarme, ¿qué es lo que quiero resaltar? ¿Sobre que quiero hacer hincapié? Estas dos preguntas tuvieron su pronta respuesta. Lo que pretendo subrayar es el don de la ubicuidad de la autoestima. Me percaté que esa era la idea que subyacía en mi mente cuando escribí la primera parte. Así como para Freud los instintos y sus vicisitudes ocupaban el centro de la escena en la problemática psicológica yo había llegado a considerar a la autoestima y sus trastornos como un común denominador en el campo de la clínica. Dichos trastornos pueden ser centrales o periféricos, graves o moderados, agudos o crónicos, de larga data o más recientes, pero siempre están presentes. Es un hilo conductor que atraviesa a todos mis pacientes (y también me incluyo). Podría decirse que no existe algo así como una persona con la autoestima perfecta. Es una característica de la personalidad humana que, debido a todos los ingredientes que requiere para forjarse de manera saludable como lo señalaba en el primer trabajo, lleva a que sea prácticamente imposible no hallar algún déficit, alguna disfuncionalidad en su construcción. Al decir esto no subestimo para nada otros factores patógenos. No ignoro la importancia del conflicto, de los hechos traumáticos en la historia personal del individuo ni el rol enfermante de ciertas privaciones. Nada de eso. Lo que sí quiero enfatizar es que el contexto en el que se despliegan estos factores es en el de una persona con una autoestima más o menos comprometida. Y, por esta razón fundamental, es menester a mi juicio contar dentro de nuestro arsenal terapéutico con un conocimiento sólido de sus vicisitudes.

La psicología pulsional, hoy por hoy, se ha constituido en una rémora para la adquisición de nuevos conocimientos atinentes a estas cuestiones teóricas de gran importancia práctica en la clínica. En la formación como psicoanalistas estas concepciones perimidas, desafortunadamente, siguen ocupando demasiado espacio y no dejan el lugar que ameritan estas nuevas y eficaces herramientas. Se sigue machacando sobre la importancia central del Complejo de Edipo, sobre las fijaciones en las etapas psicosexuales del desarrollo, sobre la libido homosexual y no se atiende ni se entiende los distintos contextos intersubjetivos que dan lugar a la psicopatología, elemento clave que distingue la perspectiva relacional en psicoanálisis.

Para ilustrar estos matices pensé que lo más adecuado era nutrirme de la clínica y para ello citaré distintos ejemplos tomados de mis pacientes. 


\section{Mi paciente Gabriel.}

Gabriel es un ingeniero de unos treinta y pico de años que me consultó hace unos meses. Había tenido en su poder mi número de teléfono hace ya algunos años y, finalmente, como suele suceder, envuelto en una situación desesperante, se había decidido a consultarme. Se sentía ansioso, deprimido, no podía conciliar el sueño y solo sobrevivía a base de psicofármacos. Se sentía fracasado en su trabajo profesional y en su vida afectiva. Ahora hacía poco que estaba saliendo con una mujer que realmente le gustaba ya que sus otras experiencias previas (aclaremos que el paciente era soltero) después de un tiempo le aburrían. La crisis que lo había empujado a la consulta había sido gatillada por un distanciamiento de la pareja. La mujer no contestaba sus mensajes y esto suscitaba en mi paciente un dolor y una angustia indescriptible.

Con este panorama ya pude figurarme donde le apretaba el zapato a este hombre. Sí, acertaron. Tenía un problema con su autoestima. ¿Qué me llevó a pensar esto? Una decepción amorosa suele ser un disparador muy comúnmente hallado en estos desequilibrios psicológicos. Un paciente ganado en la apreciación de si mismo por convicciones invalidantes va a tender a vivir cualquier desengaño o frustración en una relación con una persona que le importa de una manera catastrófica. Lo va a tomar como una confirmación incuestionable de su insuficiencia, de su defectuosidad, de estar irremisiblemente signado por la mala suerte. Desde esta perspectiva no hay lugar en su mente para considerar las dificultades que tiene la otra parte con comprometerse emocionalmente. No señor, todo es culpa suya, culpa de su mala fortuna y de su insignificancia. La elección de una persona con estas limitaciones afectivas tampoco es azarosa. Se la elige porqué precisamente es así. Lo que tiende a generar atracción son precisamente estas actitudes rechazantes. Recordemos lo que en otra parte he dado en llamar "el síndrome de Groucho Marx": "No sería socio de un club que me aceptara a mí como socio".

Teniendo muy en cuenta la advertencia de Paul Wachtel acerca de cómo los psicoterapeutas tienden a prestarle poca atención a las vicisitudes de la vida cotidiana de los pacientes, todas las comprensiones arriba mencionados las incluyo en las explicaciones que le proporciono a mis pacientes acerca de la dinámica de sus interacciones con sus respectivas parejas. Y esta estrategia me ha llevado una y otra vez a comprobar que John Bowlby tenía razón cuando afirmaba que no hay nada más tranquilizante que una buena explicación. Uno le aporta al paciente una perspectiva distinta de por qué le pasa lo que le pasa. De porqué se siente como se siente. $Y$ eso produce varios efectos benéficos. En primer lugar, les produce mucho alivio, en segundo lugar, esta misma comprensión pone 
en marcha un proceso de curación de su autoestima. ¡Es muy bueno sentirse comprendido y no ser recriminado! Hay que tener en cuenta que las personas que están en una situación análoga a la de mi paciente Gabriel, suelen responder a una frustración como la que describíamos con dos reacciones prototípicas y las dos acarrean consecuencias dañinas. $O$ se deprimen y entran en un estado melancólico o si no, como expresión de su amor propio herido, reaccionan con violencia, que va desde romper la relación a apelar a la violencia física. El entender esta dinámica con nuestra ayuda les sirve para ir encontrando, también con nuestro auxilio, formas diferentes y mucho más constructivas de expresar un reclamo, una protesta o una disconformidad sin recurrir a actitudes extremas. Si hay algo que no podemos subestimar es la tentación, por parte del paciente, de suponer que todo este sufrimiento al que se ven expuestos cuando están en una relación con alguien que les importa, se resolvería si evitaran comprometerse. Por supuesto, e imagino que lo estarán adivinando, este es un tema que va a surgir una y otra vez en la transferencia con el analista. ¿El analista nos está llevando por el buen camino o se opone a nuestras tentaciones de soluciones drásticas debido a su insensibilidad? Nuevamente entender la dinámica de estos desencuentros irá permitiendo consolidar la relación con el paciente y además irá ayudando a la consolidación progresiva de sus relaciones extra-terapeúticas.

Para Gabriel le resultó muy revelador tomar conciencia de cómo esta susceptibilidad exagerada y su consiguiente tendencia a interpretar en forma catastrófica su responsabilidad en las vicisitudes de su desempeño profesional también afectaba los resultados que obtenía. Paulatinamente la elaboración de este paralelismo fue haciendo que fuera obteniendo mejores logros en el área laboral.

Como lo indicaba en mi primer trabajo, es fundamental preguntarnos y reconstruir con el paciente el contexto intersubjetivo en el que fraguó esta autoestima deficitaria. En este caso, lo que sobresalía era una madre que había sido y seguía siendo extremadamente temerosa. Vivía imaginando peligros por doquier. Las calles eran peligrosas, salir era peligroso, el clima era peligroso, en fin, vivir era peligroso. Vivía anticipando toda clase de catástrofes que podían ocurrir. Este es el clima emocional en el que crecieron mi paciente y sus dos hermanos. Sabemos cuales son las consecuencias de esta actitud en los hijos. Erosiona la autoestima, genera dudas profundas con respecto a los recursos con los cuales contamos y contribuye decisivamente a que tendamos a experimentar las contrariedades y dificultades con las que topamos en la vida como gigantescas y tremendas, corroborando así lo que nos advertía nuestro progenitor. La inseguridad que estas convicciones invalidantes promueve trae aparejado que, debido a las expectativas negativas que acompañan nuestras iniciativas, las posibilidades de obtener malos resultados se incrementen. Esto a su vez tiende a reforzar la creencia de que estamos signados por la 
mala suerte y que somos perdedores natos. Explicar esta dinámica siniestra es una herramienta esencial para rescatar a nuestros pacientes de estos círculos viciosos, tarea que es muy liberadora y gratificante para el paciente y para el terapeuta.

\section{El caso de Ricardo}

Ricardo, un empleado bancario de unos 40 años, viene a la consulta quejándose de ansiedad, insatisfacción crónica en su vida personal y profesional y síntomas depresivos. Está separado de su mujer, y tiene dos hijos de ese matrimonio, una nena de doce y un varoncito de 9 años. Todavía no ha efectivizado su divorcio y no ha hecho por tanto una separación de bienes. El motivo aparente que había motorizado la separación hace ya algunos años, había sido una infidelidad de la esposa, aunque las cosas entre ellos hacía rato que venían mal. Cuando empezó su terapia estaba saliendo con una mujer que conocía de su pueblo natal, donde ella vivía. Mi paciente viajaba regularmente a este pueblo rural, ubicado a unos $300 \mathrm{~km}$. de Buenos Aires. Esta relación se había vuelto también insatisfactoria, fundamentalmente por los celos obsesivos de esta mujer y los consiguientes reclamos y recriminaciones. Sin embargo, Ricardo dudaba de la validez de lo que sentía y por tanto oscilaba entre una actitud pasiva y reacciones de rabia explosiva.

Con esta sucinta presentación, veamos como mis comprensiones del papel de la autoestima en la génesis de nuestras dificultades me permitió orientarme. En primer lugar, aparecía algo que uno tiende a observar como algo recurrente en estas personas, a saber, la propensión a establecer relaciones afectivas predominantemente insatisfactorias. Y digo predominantemente, porque siempre hay aspectos rescatables y valorables. Por lo tanto hay que estar advertido de no ser simplista y dar por sentado que la cuestión se resolvería terminando la relación. De lo que indudablemente el paciente debe irse soltando es un modelo que tiene la "virtud" de perpetuar el sufrimiento. Es menester señalarle que sus actitudes disfuncionales que pone en juego para expresar su disconformidad también colaboran y mucho en la configuración de sus padecimientos y en la evitación de escenarios más gratificantes. Otro tema recurrente es la dificultad notoria en exponer con firmeza autoafirmativa sus deseos, intereses y también sus disconformidades. Las convicciones invalidantes de inferioridad, insuficiencia e inadecuación, los hace sentirse sin derechos, sin merecimientos y por lo tanto expuestos a ser desechados a la primera de cambios. Esto, como comprenderán, aumenta crónicamente su sentimiento de infelicidad y refuerza la convicción depresiva de sentirse condenados por la mala suerte.

También con Ricardo, la progresiva elaboración de estos temas, fue cambiando el panorama. Donde antes estaba la resignación depresiva, empezó a surgir la esperanza. Se 
empezó a sentir de mejor humor y, como suele ocurrir, le empezaron a suceder cosas buenas. Una relación más satisfactoria con una mujer compañera de trabajo, unas vacaciones con sus hijos diferentes y divertidas, una actitud más aplomada en el trato con la exmujer. Ricardo sigue su tratamiento y evoluciona favorablemente.

\section{El caso de Francisco}

Francisco es un abogado soltero de unos treinta años. Me consulta por sentirse deprimido, infeliz, envuelto en una desagradable sensación de sentirse desahuciado, sin salida. Hace ya un tiempo que sale con una mujer casi veinte años mayor que él, con la que ha establecido una relación en la cual él es sistemáticamente maltratado y ninguneado. Salvo ocasionales momentos de pasión amorosa la desdicha y el maltrato es la regla. Al indagar sobre su historia personal, surgen varios antecedentes previos de relaciones organizadas bajo el mismo patrón: un noviazgo y convivencia previo con una mujer que se tornó un calvario, un empleo previo en el estudio de una tía abogada donde, durante varios años fue descalificado y postergado, un trabajo actual en relación de dependencia que siente rutinario y mal remunerado y así. ¿Les suena lo que conté hasta ahora? Efectivamente, se reiteran temas y dinámicas que he venido señalando en los anteriores ejemplos. ¿Y en cuanto el contexto intersubjetivo que los precede? Nuevamente nos encontramos con una madre extremadamente temerosa y un padre que fue históricamente devaluado y maltratado. Hasta acá no hay nada novedoso. Pero este caso me da pie para ilustrar otro aspecto llamativo de estas historias y es el siguiente: en los tres casos que menciono y en otros más he encontrado regularmente la presencia en el psiquismo de los pacientes de un introyecto persecutorio, que, como una voz interna tiende a convertirse en el representante de esta descalificación al self del paciente, abrumándolo con desprecio, insultos y burlas. No sólo eso, sino que constantemente lo vive intimidando con pronósticos ominosos sobre su futuro. Es de esta especie de energúmeno, como suelo denominarlo, del cual emanan también ataques furibundos contra el analista, sembrando dudas acerca de sus verdaderas intenciones.

Es importante tenerlo muy presente porqué exponer a este personaje a la luz (lo que he dado en llamar el efecto Drácula) y someter a la reflexión consciente su prédica destructiva y los argumentos falaces que emplea es una herramienta terapéutica fundamental. Tal como ocurre en la leyenda del conde Drácula, iluminar a este engendro lo debilita, le va quitando poder y esto se traduce en mejoría. Es como si fuera un hechizo que se va evaporando. 
Francisco pudo conseguir una beca por la que hace tiempo venía bregando infructuosamente, terminó su relación insatisfactoria con la mujer con la que salía y empezó otra con una mujer de su edad que lo quiere y respeta, terminó la relación con un colega deshonesto, se empezó a desempeñar con idoneidad y soltura en algunos casos que tenía de su práctica privada, con la consiguiente mejora en su economía personal y hoy por hoy está organizando un viaje de placer a EEUU. Pero tengamos presente, para no caer en simplificaciones, que como dice el dicho "la cabra tira al monte". Es decir, la añoranza por el viejo modelo sigue presente y hay que tenerla muy en cuenta. Especialmente quiero referirme acá a un factor poco mencionado, pero no por ello menos importante a considerar. $Y$ es el hecho que, muchas veces, estas personas relatan que en esas relaciones insatisfactorias encuentran una alta dosis de placer en el sexo, mientras que, en las otras, más satisfactorias desde el punto de vista del trato, no. Es un tema que es menester prestarle atención, porque se constituye en algo que tiende a robustecer la nostalgia por lo malo y conocido.

Cuando le leí esto que había escrito al paciente, Francisco me dijo que le parecía bien, pero insistió que no dejara de incluir algo importante. Se refería a lo que el describió como un clic que se le produjo en su cabeza cuando pudo dejar de esperar de esa mujer que lo quisiera, que lo aceptara. Decía que perder esa condición de vida o muerte que adquiría para él ese hecho, lo había relajado muchísimo.

El convencimiento de que es posible vivir de otra manera, más plena, más satisfactoria, en una persona que por años se ha visto a sí mismo como alguien signado por la desgracia, no se consolida de la noche a la mañana. Pero decir esto de ninguna manera borra el hecho, de que estar equipado con estas comprensiones acerca de las vicisitudes de la autoestima, es una brújula inestimable con la cual contamos para guiar eficazmente nuestros esfuerzos terapéuticos.

\section{Reflexiones finales}

A guisa de homenaje quisiera empezar esta última parte con dos frases de John Bowlby que interpretan de manera cabal mi pensamiento psicoanalítico. La primera es cuando afirma que "No hay nada más práctico que una buena teoría" y la segunda "Cuanto mayor sea el conocimiento que un analista tiene de las situaciones infantiles que pueden conducir a un desarrollo perturbado, mejor podrá entender y ayudar a sus pacientes." Son frases expresadas de una manera sencilla pero ¡cuánta sabiduría encierran! 
Inspirado por esta filosofía voy a tratar de brindar un pantallazo de los ítems que hay que tener en cuenta en nuestras aproximaciones comprensivas: 1) Tener presente la ubicuidad de los trastornos de la autoestima; 2 ) prestar atención a los indicadores que dan cuenta de esos trastornos, tanto como a las convicciones irracionales de defectuosidad e inferioridad que las sustentan; 3 ) tener presente $y$, cuando sea oportuno, explorar los distintos contextos intersubjetivos pasados y presentes que han contribuido a forjar dicha sintomatología; 4) tener "in mente", como espero haber ilustrado suficientemente aquí, el carácter recurrente de ciertas configuraciones relacionales y por último, 5) recordar que el progreso no es de una vez y para siempre. Indefectiblemente va a haber marchas y contramarchas, incursiones en el nuevo escenario y retrocesos a lo conocido, pero en la medida que se van obteniendo buenos resultados, cada vez la atracción por lo viejo se va debilitando.

\section{REFERENCIAS}

Arendar, R. (2010). ¿Hundirse con el Titanic? Clínica e Investigación Relacional, 4 (1): 236-242. Arendar, R. (2014). La autoestima y sus vicisitudes. Clínica e Investigación Relacional, 8 (3): 394402.

Bowlby, J. (1989). Una Base Segura (Aplicaciones clínicas de una teoría del apego) Barcelona: Editorial Paidós SAICF

Wachtel, P. (2014). Cyclical Psychodynamics and the Contextual Self: The inner World, the Intimate World, and the World of Culture and Society. New York: Routledge.

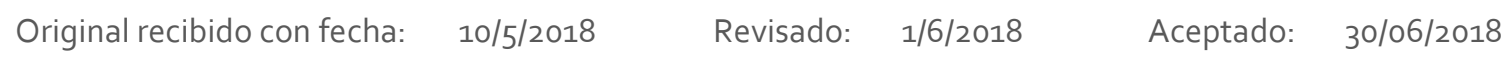

